of data. For example, error messages would appear if appointments were double booked. We also had a built-in follow up system. This would highlight patients whose results hadn't been followed up which would also help prevent mistakes. This QI project had 5 cycles in which the system was finetuned, tutorials were given to members of the MDT and feedback was taken. This helped us promote a sustainable change and allowed us to troubleshoot any issues with the appointment system. After each cycle our objectives were measured quantitatively.

Results

1. We found that the number of patients with three pieces of patient identifiable data increased by $56 \%$ (patients with at least 2 pieces of identifiable details also increased).

2. The number of patients with clear reasons inputted for coming to the paediatric ambulatory care unit increased by $22 \%$.

3. The number of double bookings decreased by $20 \%$.

4. The number of patients who weren't followed up were also reduced (results went down from 4 serious incident form in the 6 month period before the computerised system to no serious incident forms in the 6 months after the system.

Conclusions This QI project showed that simple IT solutions can often lead to dramatic improvements in patient safety and better care. The reduction of human error is important for any paediatric department. Involving the multidisciplinary team in projects can lead to a more sustainable change and is necessary when creating changes to systems.

\section{PARENTAL PERCEPTION ON INHALER AND SMOKING AT HOME}

Sze Ka Ng, Sze Ka Ng, Florence Choi, Po King Ma. Hong Kong

\subsection{6/bmjpo-2021-RCPCH.155}

Background Fear of treatment of asthma is common amongst patients and their carers, and often leads to poor disease control and poor quality of life. Some carers preferred the use of complementary or alternative medicine, such as Chinese herbal medicine or supplement, to inhaled medications.

Objectives This paper aims to describe the perception of parents with asthmatic children on inhaler treatment and smoking at home.

Methods The clinical trial was carried out at an acute respiratory Paediatric unit in a public hospital in Hong Kong. The proposal of the clinical trial was registered in the Chinese Clinical Trial Registry, World Health Organization Organisation (ChiCTR1800019706) and was approval from the Ethic Committee review board was obtained parental consents were obtained. Fifty-six children aged 4-11 years were admitted to an acute paediatric unit for asthmatic attack during the study period from Oct 2018 to Jun 2019. Their parents were invited to participate in a semi-structured interview using open-ended questions, guided by several themes. The interview lasted 15 to 20 minutes. Content analysis was used to analyze the data collected from the interviews. All transcriptions were undertaken by the same investigator.

Results Four aspects were concluded.

Doubt about asthma diagnosis. According to the most updated concept, asthma is considered an allergic disease that can be controlled without primary symptoms, such as repeated coughing, wheezing and breathing difficulties.
However, many parents perceived asthma as a different entity from allergic airway. Some were unable to differentiate between asthmatic attack, upper respiratory tract infection and wheezing in pneumonia. With these misconceptions, parents would doubt the diagnosis of asthma and would default follow-up eventually.

Steroid phobia. Many parents expressed their concern on the adverse effects of corticosteroids on their children, especially for those who started treatment at an older age. They were worried that inhaled corticosteroids would do more harm than good. One parent even used the term 'steroid abuse' when referring to the regular use of steroid. Another parent had fear of oral ulcer development as a side effect of corticosteroid.

Perception on inhaler. There were several misconceptions on inhaler medications. Some parents perceived the long term use of bronchodilator would lead to asthma or persist the asthma symptoms. Some thought nebulizing medications were more effective than delivering medications via inhalers.

Smoking at home. Some parents believed indirect exposure to tobacco smoke at home would not affect the control of asthma in their children. Parents understood the harm of smoking at home but expressed their difficulty in asking the elder family member living with child to quit smoking.

Conclusions These qualitative data highlight the communication problem between healthcare professionals and patients and the misconceptions of asthma management. Firstly, the updated concept on allergic diseases such as asthma, was not well-publicized. Many parents could not accept their children being diagnosed with asthma, although they themselves had asthma or other allergic disease during their childhood. Furthermore, the concept of controlled asthma used by healthcare professionals and parents are often different. Lastly, third-hand smoking is a commonly overlooked issue.

\section{HEALTH-RELATED QUALITY OF LIFE OF RARE DISEASE PATIENTS AND CARE-GIVERS IN HONG KONG}

Yvette Nga Chung Ng, Brian Hon Yin Chung, Claudia Ching Yan Chung. Hong Kong

\subsection{6/bmjpo-2021-RCPCH.156}

Background A rare disease (RD) is a disease affecting less than one in 2,000 people. In Hong Kong, one in 67 people is living with one or more RDs. RD patients live with different degrees of life-long physical or intellectual disability and often require special care. Approximately $80 \%$ of the RDs are genetic in origin, in which some are with childhood onset. The paediatric RD patients constitute $35 \%$ of the whole $\mathrm{RD}$ population in Hong Kong.

Objectives To investigate the health-related quality of life (HRQoL) of patients and care-givers in the RD population in Hong Kong.

Methods This was a cross-sectional study between 25 April and 16 October 2020. The EuroQol 5-Dimension 3-Level (EQ-5D-3L), a generic preference-based patient-reported outcome measure instrument, was distributed to the RD patients and care-givers through $\mathrm{RD}$ patient groups to describe HRQoL. The five dimensions assessed were mobility, self-care, usual activity, anxiety/depression and pain/discomfort. The three levels represent severity in each dimension, with Level 1 indicating absence of problem and Level 3 indicating extreme 